\title{
GRAVITY-DRIVEN ACCELERATION AND KINETIC INFLATION IN NONCOMMUTATIVE BRANS-DICKE SETTING
}

\author{
S.M.M. Rasouli ${ }^{1,2,3}$, Paulo Vargas Moniz ${ }^{1,2}$ \\ ${ }^{1}$ Departamento de Física, Universidade da Beira Interior, \\ Rua Marquês d'Avila e Bolama, 6200 Covilhã, Portugal, \\ mrasouli@ubi.pt,pmoniz@ubi.pt \\ ${ }^{2}$ Centro de Matemática e Aplicações (CMA - UBI), Universidade da Beira Interior, \\ Rua Marquês d'Avila e Bolama, 6200 Covilhã, Portugal \\ ${ }^{3}$ Physics Group, Qazvin Branch, Islamic Azad University, Qazvin, Iran
}

\begin{abstract}
By assuming the spatially flat FLRW line-element and employing the Hamiltonian formalism, a noncommutative (NC) setting of the Brans-Dicke (BD) theory is introduced. We investigate gravity-driven acceleration and kinetic inflation in this NC BD cosmology. Despite to the commutative case, in which both the scale factor and BD scalar field are obtained in power-law forms (in terms of the cosmic time), in our herein NC model, we see that the power-law scalar factor is multiplied by a dynamical exponential warp factor. This warp factor depends on not only the noncommutative parameter but also the momentum conjugate associated to the BD scalar field. For very small values of this parameter, we obtain an appropriate inflationary solution, which can overcome problems within standard BD cosmology in a more efficient manner. Moreover, we see that a graceful exit from an early acceleration epoch towards a decelerating radiation epoch is provided. For late times, due to the presence of the NC parameter, we obtain a zero acceleration epoch, which can be interpreted as the coarse-grained explanation.
\end{abstract}

\section{Introduction}

In the BD setting employed in $[1,2]$, a variable $\mathrm{BD}$ coupling parameter rather than a constant one has been supposed. Then, without introducing any scalar potential or cosmological constant, an accelerated expanding universe, from the kinetic energy density of a dynamical Planck mass, has been obtained. In [2], it has been argued that, to meet sufficient inflation, the scale factor in the Einstein frame must accelerate. However, there is no source to get an accelerating scale factor in that frame. In other words, in the commutative case of the BD theory [3] (in the Jordan frame), even by assuming variable $\omega$, there is a fundamental problem with kinetic inflation: regardless of the form of $\omega(\phi)$, all the $\mathrm{D}$ branch $^{1}$ solutions are encountered with the graceful exit problem [2].

In our herein $\mathrm{NC}$ BD setting ${ }^{2}$, which, regardless of varying $\omega$, can be considered as a generalized set up of [2], we will obtain an accelerating scale factor for the early Universe, without encountering the abovementioned problems of [2]. Moreover, we will show that requirements of an inflationary epoch (specially, the nominal as well as sufficient conditions) are satisfied in a more convenient manner when the noncommutativity parameter is present.

In this paper, by assuming a spatially flat FLRW universe, a generalized BD theory, and introducing a particular NC Poisson bracket between the BD scalar field and the logarithm of scale factor, we review the solutions (associated to the NC equations of motion) and discuss the effects of noncommutativity.

\section{Noncommutative Brans-Dicke Cosmologi- cal Setting}

We start with the spatially flat FLRW metric as the background geometry, namely

$$
d s^{2}=-N^{2}(t) d t^{2}+e^{2 \alpha(t)}\left(d x^{2}+d y^{2}+d z^{2}\right),
$$

where $a(t)=e^{\alpha(t)}$ is the scale factor and $N(t)$ is a lapse function.

To get a general set of the field equations, let us start with the Lagrangian associated to the (generalized ${ }^{3}$ ) BD theory in the Jordan frame as

$$
\mathcal{L}[g, \phi]=\sqrt{-g}\left[\phi R-\frac{\omega(\phi)}{\phi} g^{\mu \nu} \nabla_{\mu} \phi \nabla_{\nu} \phi-V(\phi)\right](2)
$$

\footnotetext{
${ }^{1}$ We will introduce the $\mathrm{D}$ and $\mathrm{X}$ branches later.

${ }^{2}$ Most of the discussions of this work has been reported in our previous paper [4].

${ }^{3}$ It has been recently shown $[5,6]$ that, instead of adding a scalar potential to $\mathrm{BD}$ action, such a scalar potential can be induced from the geometry of an extra dimension.
} 


$$
+\sqrt{-g} \mathcal{L}_{\text {matt }}
$$

where $\mathcal{L}_{\text {matt }}=16 \pi \rho(\alpha)$ is the Lagrangian density of ordinary matter ( $\rho$ is the energy density), which does not explicitly depend on the BD scalar field $\phi$. The BD parameter $\omega(\phi)$ is a function of the BD scalar field and varies with the space-time point; $V(\phi)$ is the scalar potential, the greek indices run from zero to 3 and $R$ is the Ricci scalar associated to the metric $g_{\mu \nu}$, whose determinant is denoted by $g$. Therefore, the Hamiltonian of the model is given by

$$
\begin{aligned}
\mathcal{H} & =-\frac{N e^{-3 \alpha}}{2 \chi \phi}\left[\frac{\omega(\phi)}{6} P_{\alpha}^{2}-\phi^{2} P_{\phi}^{2}+\phi P_{\alpha} P_{\phi}\right] \\
& +N e^{3 \alpha}(V-16 \pi \rho),
\end{aligned}
$$

where $\chi \equiv 2 \omega+3$, and $P_{\alpha}, P_{\phi}$ are the conjugate momenta associated to $\alpha$ and $\phi$, respectively. In this work, we work with the comoving gauge, namely, we set $N=1$. Instead of the commutative phase space where the Poisson algebra is given by $\{\alpha, \phi\}=0$, $\left\{P_{\alpha}, P_{\phi}\right\}=0,\left\{\alpha, P_{\alpha}\right\}=1$ and $\left\{\phi, P_{\phi}\right\}=1$, to investigate the effects of a classical evolution of the noncommutativity on the cosmological equations of motion, we presume the following Poisson commutation relations between the variables as $\{\alpha, \phi\}=\theta,\left\{P_{\alpha}, P_{\phi}\right\}=0$, $\left\{\alpha, P_{\alpha}\right\}=1$ and $\left\{\phi, P_{\phi}\right\}=1$ where the NC parameter $\theta$ is taken as a constant. Employing these noncommutation relations and the Hamiltonian (3) leads us to deformed equations of motion as

$$
\begin{aligned}
\dot{\alpha} & =-\frac{e^{-3 \alpha}}{2 \chi \phi}\left[\frac{\omega(\phi)}{3} P_{\alpha}+\phi P_{\phi}\right] \\
& -\frac{\theta e^{-3 \alpha}}{2 \chi \phi}\left[\frac{1}{6} \frac{d \omega(\phi)}{d \phi} P_{\alpha}^{2}-2 \phi P_{\phi}^{2}+P_{\alpha} P_{\phi}\right] \\
& +\frac{\theta e^{3 \alpha}}{\phi}\left[V(\phi)+\phi \frac{d V(\phi)}{d \phi}-16 \pi \rho\right], \\
\dot{P}_{\alpha} & =e^{3 \alpha}\left[-6 V(\phi)+16 \pi\left(6 \rho+\frac{d \rho}{d \alpha}\right)\right], \\
\dot{\phi} & =-\frac{e^{-3 \alpha}}{2 \chi}\left(P_{\alpha}-2 \phi P_{\phi}\right) \\
& -\theta e^{3 \alpha}\left[6 V(\phi)-16 \pi\left(6 \rho+\frac{d \rho}{d \alpha}\right)\right] . \\
\dot{P}_{\phi} & =\frac{e^{-3 \alpha}}{2 \chi \phi}\left[\left(P_{\alpha}-2 \phi P_{\phi}\right) P_{\phi}+\frac{1}{6} P_{\alpha}^{2} \frac{d \omega(\phi)}{d \phi}\right] \\
& -\frac{e^{3 \alpha}}{\phi}\left[V(\phi)+\phi \frac{d V(\phi)}{d \phi}-16 \pi \rho\right],
\end{aligned}
$$

where a dot denotes the differentiation with respect to the cosmic time and we have assumed $\phi=\phi(t)$. We should note that the equations of motion associated to the momenta $P_{\alpha}$ and $P_{\phi}$, under the proposed NC deformation, are the same as ones in the commutative case and, obviously, in the limit $\theta \rightarrow 0$, Eqs. (4) and (6) are reduced to the corresponding standard commutative equations.
In what follows, we investigate the cosmological implications of this NC BD setting for a very simple case in which the BD parameter is a constant and the scalar potential and the ordinary matter are absent.

\section{Gravity-Driven acceleration for cosmolog- ical models in the noncommutative BD Setting}

As mentioned, we would like to assume a very simple case in which we set $\omega(\phi)=\omega=$ constant, $\rho=0$ and $V(\phi)=0$. Therefore, from (5), we get $\dot{P}_{\alpha}=0$, which gives a constant of motion. Thus, we get $P_{\alpha}=c_{1}$; also, Eqs. (4) and (7) give $P_{\phi}=c_{2}^{ \pm} \phi^{-1}$ where $c_{1}$ and $c_{2}^{ \pm} \neq 0$ are the integration constants. These constants are not independent; by substituting them into the Hamiltonian constraint, we obtain $c_{1}^{ \pm}=\left(3\left|c_{2}\right| / \omega\right)\left[-\operatorname{sgn}\left(c_{2}\right) \pm \xi\right]$ where $\xi \equiv \sqrt{\chi / 3}, \omega \neq 0$ and $\operatorname{sgn}(x)=x /|x|$ is the signum function. Thus, from (6), $\dot{\phi}$ can be given by

$$
\dot{\phi}=-\frac{f^{ \pm}}{\xi a^{3}} \quad \text { where } \quad f^{ \pm} \equiv \frac{\left|c_{2}\right|}{2 \omega}\left[-\operatorname{sgn}\left(c_{2}\right) \xi \pm 1\right] .
$$

By employing the obtained expressions associated to the momenta and the integration constants, from Eqs. (4) and (6) we obtain

$$
H=h^{ \pm}\left(\frac{\dot{\phi}}{\phi}\right) \quad \text { where } \quad h^{ \pm} \equiv g^{ \pm}+\frac{c_{2} \theta}{\phi},
$$

where $H=\dot{a} / a$ is the Hubble constant and $g^{ \pm} \equiv$ $-\frac{1}{2}\left[1 \pm \operatorname{sgn}\left(c_{2}\right) \xi\right]$. We should note that the above equations corresponding with each sign of ${ }^{4} c_{2}$ yield two branches for the Hubble parameter. If we assume an attractive gravity [7], i.e., $\phi>0$, the values of $\dot{\phi}$ as well as $h$ corresponding to each branch ${ }^{5}$ must be specified to get an expansion or contraction universes. For instance, in a special case, by setting $c_{2}>0$ and $\theta=0$, we obtain $H=-\frac{\dot{\phi}}{2 \phi}(1 \pm \xi)$. In this case, for $\xi<1$, $H>0$ only when $\dot{\phi}<0$, and $H<0$ only when $\dot{\phi}>0$ (for both of the branches). While, for $\xi>1$, to have a positive Hubble expansion we must choose the upper sign for $\dot{\phi}<0$ and the lower sign for $\dot{\phi}>0$.

Now, let us investigate a general case. We get the acceleration of the scale factor as $\frac{\ddot{a}}{a}=$ $-\frac{1}{6 \phi}\left[\rho^{(\phi)}+3 p^{(\phi)}\right]=-\left(\frac{\dot{\phi}}{\phi}\right)^{2}\left(2 h^{2}+h+\frac{c_{2} \theta}{\phi}\right)$, where

\footnotetext{
${ }^{4}$ For simplicity of expressing the quantities, we will sometimes drop the index \pm .

${ }^{5}$ Following $[8,1,2]$, we will call the branches as follows. As in the Einstein frame, one of the branches always yields an expanding universe, while the other gives a contracting universe. Therefore, the solutions correspond to the former, and the latter are called the $\mathrm{X}$ branch and $\mathrm{D}$ branch, respectively. In our herein model, when $c_{2}>0, \mathrm{X}$ branch and $\mathrm{D}$ branch solutions correspond to the upper sign and lower sign solutions, respectively. When $c_{2}<0$, we note the transformations derived after Eq. (16).
} 
the energy density and pressure associated to the BD scalar field are given by [6]

$$
\begin{aligned}
\rho^{(\phi)} & \equiv-T_{0}^{0(\phi)}=3 h^{2}\left(\frac{\dot{\phi}^{2}}{\phi}\right), \\
p^{(\phi)} & \equiv T_{i}^{i(\phi)}=\left(3 h^{2}+2 h+\frac{2 c_{2} \theta}{\phi}\right)\left(\frac{\dot{\phi}^{2}}{\phi}\right),
\end{aligned}
$$

where $i=1,2,3$ (with no sum) and we have employed relations (8) and (9). Therefore, in order to get an accelerating universe, the following constraint must be satisfied

$$
2 h^{2}+h+\frac{c_{2} \theta}{\phi}<0
$$

As $\rho^{(\phi)}>0$, relation (11) and constraint (12) dictate that the pressure must take negative values.

From (9), we get a relation between the scale factor and the BD scalar field as

$$
a(t)=a_{i}[\phi(t)]^{g} e^{-c_{2} \theta \phi^{-1}},
$$

where $a_{\mathrm{i}}=e^{\alpha_{\mathrm{i}}}$ is an integration constant, which corresponds to $\alpha$ in a specific time. Eq. (13) indicates that the NC parameter appears in the power of an exponential warp factor. We can easily show that this time-dependent warp factor appears in the differential equation associated to $\phi$ [see Eq. (14)] and makes it a very complicated differential equation, such that we have to solve it numerically instead. Employing Eqs. (13) and (8), we get a differential equation for the BD scalar field as

$$
\dot{\phi} \phi^{3 g} e^{-3 c_{2} \theta \phi^{-1}}=-\frac{f}{a_{i}^{3} \xi},
$$

where, according to (8), $f$ depends on $c_{2}$ and $\omega$.

In the commutative case, dependent on the value of $\omega$, we obtain two different types of solutions: (i) when $g=-1 / 3$ (or $\omega=-4 / 3$ ), which corresponds to both the lower sign (when $c_{2}>0$ ) and the upper sign (when $\left.c_{2}<0\right)$, the solutions correspond to the de Sitter-like space as $a(t)=a_{\mathrm{i}} \phi_{\mathrm{i}}^{-\frac{1}{3}} e^{m t}$ and $\phi(t)=\phi_{\mathrm{i}} e^{-3 m t}$, where $\phi_{\mathrm{i}}$ is an integration constant, and $m \equiv \frac{-\left|c_{2}\right|}{8 a_{i}^{3}}\left[-\operatorname{sgn}\left(c_{2}\right) \pm 3\right]$, ii) for $\omega \neq-4 / 3$, we obtain the generalized version of the well-known O'Hanlon-Tupper solution [9, 7] as $a(t)=\tilde{a}_{\mathrm{i}}\left(t-t_{\mathrm{ini}}\right)^{r_{ \pm}}$and $\phi(t)=\tilde{\phi}_{\mathrm{i}}\left(t-t_{\mathrm{ini}}\right)^{s_{ \pm}}$with

$$
\begin{aligned}
& \tilde{\phi}_{\mathrm{i}}=\left\{\frac{\left|c_{2}\right|}{2 a_{\mathrm{i}}^{3} \omega}\left[\operatorname{sgn}\left(c_{2}\right) \mp \frac{(\omega+1)}{\xi}\right]\right\}^{s_{ \pm}}, \\
& \tilde{a}_{\mathrm{i}}=a_{\mathrm{i}} \tilde{\phi}_{\mathrm{i}}^{g}=a_{\mathrm{i}}\left\{\frac{\left|c_{2}\right|}{2 a_{\mathrm{i}}^{3} \omega}\left[\operatorname{sgn}\left(c_{2}\right) \mp \frac{(\omega+1)}{\xi}\right]\right\}^{r_{ \pm}},
\end{aligned}
$$

where $t_{\text {ini }}$ is an integration constant and the exponents $r_{ \pm}$and $s_{ \pm}$are given by

$$
r_{ \pm}=\frac{1}{3 \omega+4}\left[\omega+1 \pm \operatorname{sgn}\left(c_{2}\right) \xi\right]
$$

$$
s_{ \pm}=\frac{1 \mp 3 \operatorname{sgn}\left(c_{2}\right) \xi}{3 \omega+4} .
$$

Here, we should explain the role of the parameters present in the model. For a particular case where $c_{2}>0$ (or $c_{2}<0$ ), we get the solutions corresponding to $\left(r_{+}, s_{+}\right)$and $\left(r_{-}, s_{-}\right)$known as the fast and slow solutions, respectively [7].

By assuming $\omega \neq-4 / 3$ and redefining $\Phi \equiv-\ln (G \phi)$ (where $G$ is the gravitational constant), it has been shown [10] that there are duality transformations as

$$
\begin{aligned}
& \alpha \rightarrow\left(\frac{3 \omega+2}{3 \omega+4}\right) \alpha-2\left(\frac{\omega+1}{3 \omega+4}\right) \Phi, \\
& \Phi \rightarrow-\left(\frac{6}{3 \omega+4}\right) \alpha-\left(\frac{3 \omega+2}{3 \omega+4}\right) \Phi,
\end{aligned}
$$

under which the slow and fast solutions are interchanged [11], namely, $\left(r_{ \pm}, s_{ \pm}\right) \longleftrightarrow\left(r_{\mp}, s_{\mp}\right)$. However, in our model for $\theta=0$ herein, from (15), without considering the duality transformations (16), we can see that the sign of the integration constant $c_{2}$ is responsible for the mentioned role, interchanging the lowerupper solutions. More precisely, under interchanging $c_{2}>0 \leftrightarrow c_{2}<0$, the parameters $c_{1}, g$, and $f$ transform as $\left(c_{1}^{ \pm}, f^{ \pm}, g^{ \pm}\right) \longleftrightarrow\left(-c_{1}^{\mp},-f^{\mp}, g^{\mp}\right)$, and, consequently, we get $\left(r_{ \pm}, s_{ \pm}\right) \longleftrightarrow\left(r_{\mp}, s_{\mp}\right)$. Also for the general NC case, as seen from (9), the general duality transformations, not only depend on the $f, g$, and the integration constants $c_{1}$ and $c_{2}$ but also may depend on the noncommutativity parameter.

In our model, due to presence of the three parameters $c_{2}, \omega$ and $\theta$, we can obtain a good variety of solutions for $\mathrm{X}$ and $\mathrm{D}$ branches, for more detail, see [4]. Here, we are only interested in summarizing some of the results of Case Ia, namely, the lower sign with $c_{2}>0,-3 / 2<\omega<-4 / 3$ and $\theta<0$ : (i) For very small negative values for the noncommutative parameter, we have shown that the scale factor starts from a singular point at $t=0$ and increases for both of the commutative and NC cases. However, it always does not have the same behavior for these cases. For the commutative case, we always get $\ddot{a}>0$, while for the noncommutative case, for the very early times, we have $\ddot{a}>0$, but at the special point, it turns to be negative; namely, after a very small time, the phase changes and we obtain a decelerating universe. (ii) The scalar field always drops for both of the commutative and $\mathrm{NC}$ cases. (iii) The larger the integration constant $c_{2}$, the shorter the time of the accelerating phase. (iv) The smaller the $|\theta|$, the larger the slope of $a(t)$, namely $\dot{a}$. (v) In the late times, by assuming the same initial values for the parameters (except $\theta), \phi(t)$ tends to zero for both the commutative and $\mathrm{NC}$ cases. However, for the large values of the cosmic time, in the commutative case, $\ddot{a}$ never takes a constant value. While, in the NC case, we get a zero acceleration epoch. 


\section{Kinetic inflation}

In the previous section, by proposing a NC setting for the BD theory, we have mentioned that how we can overcome the graceful exit problem. However, these features alone do not guarantee a scenario for the resolution of the problems with the standard cosmology.

In this section, we first investigate the nominal condition [1] for the acceleration associated to the inflation, namely,

$$
d^{\text {Hor }}>H^{-1}, \quad \text { where } \quad d^{\text {Hor }}(t)=a(t) \int_{t_{i}}^{t} d t^{\prime} / a^{\prime} .
$$

Then, in the rest of this section, we will study the condition for sufficient inflation.

Employing Eqs. (8), (9), and after some manipulations, we obtain

$$
\frac{d \ln \left(a^{2} \phi\right)}{d t}= \pm(2 h+1)\left(\frac{\dot{\phi}}{\phi}\right) .
$$

Using (8) and integrating over $d t$, we get

$$
d^{\mathrm{Hor}}=\frac{a^{3} \phi}{|f|}-\frac{2 c_{2}}{2 g+1} d^{\mathrm{NC}}
$$

up to a constant of integration. In Eq. (19), we introduced a NC contribution of distance, $d^{\mathrm{NC}}$, as $d^{\mathrm{NC}} \equiv$ $\frac{\theta a}{c_{2}} \int P_{\phi^{\prime}}^{\prime} d t^{\prime} / a^{\prime}$. We expect that $d^{\mathrm{NC}}$ can add a positive value to the $d^{\text {Hor }}$ to properly assist in satisfying the requirement associated to the horizon problem. In order to compare, by the aid of (8), we rewrite Eq. (9) as $H=|f| h /\left(\xi a^{3} \phi\right)$. Employing this result and (19) in the nominal condition (17) yields

$$
D^{\mathrm{NC}} \equiv d^{\mathrm{Hor}}-H^{-1}=\frac{\phi a^{3}}{|f|}\left(1+\frac{\xi}{h}\right)-\frac{2 d^{\mathrm{NC}}}{2 g+1}>0 .
$$

Obviously, when $\theta$ goes to zero then $d^{\mathrm{NC}}$ vanishes, and, thus, the relation associated to the horizon distance of the commutative case is recovered. Therefore, the resulted relation is the same as corresponding one in Ref. [1] provided that the BD coupling parameter takes constant values within. Consequently, when $\theta=0$ the only acceptable result is $0<\xi<1(-3 / 2<\omega<0)$, which is obtained by choosing either the upper sign for $c_{2}>0$ or the lower sign for $c_{2}<0$.

For our herein NC case, it is important to emphasize that the $\mathrm{NC}$ parameter plays a significant role in determining whether the constraint (20) is satisfied or not. As an example, for both the commutative and the NC (case Ia) cases, in Fig. 1, we have plotted $D^{\mathrm{NC}}$ versus cosmic time. This figure shows that the constraint (20), at all times, for the NC case can be easier satisfied than its corresponding commutative case.

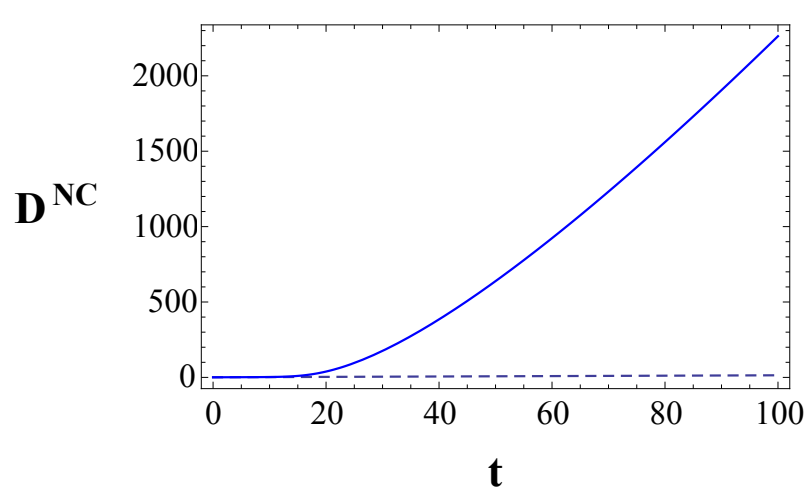

Figure 1: The behavior of the $D^{\mathrm{NC}}$, the quantity which defined as in (20), versus cosmic time. The dashed and solid curves are associated to the commutative and NC cases, respectively. This figure is plotted (as an example) to show that the nominal condition (17) for the NC solutions (particularly for the case Ia) can be easily satisfied. The initial values are $\omega=-1.4, a_{0}=$ $1=c_{2}, \theta=0$ (dashed curve) and $\theta=-0.000001$ (solid curve) [4].

In what follows, we investigate the condition for sufficient inflation, which is constrained as [2]

$$
\frac{d_{\star}^{\text {Hor }}}{a_{\star}}>\frac{1}{H_{0} a_{0}} .
$$

The quantity appeared in lhs of the above inequality is comoving size of a causally connected region at a specific earlier time $t_{\star}$. From relation (18), for the specific time $t_{\star}$, we obtain

$$
d_{\star}^{\text {Hor }}=\frac{a^{3} \phi(1-\delta)}{f \operatorname{sgn}\left(c_{2}\right)}-\left.\frac{2}{2 g+1} d^{\mathrm{NC}}\right|_{\star} .
$$

We should note that the integration constant, which was removed in relation (19), has now been included in $\delta \equiv \frac{a_{i}^{2} \phi_{i}}{a^{2} \phi}$ where the subscript $i$ stands for initial values. By assuming that $\phi>0$, we always have $\delta \geq 0$. In order to construct the inequality (21) in our herein $\mathrm{NC}$ model, which can be appropriately compared with the corresponding commutative one, we have employed a few assumptions and a lot of manipulations. Let us here just express the final result [4]:

$$
\begin{aligned}
\frac{a_{\star}^{2} \phi_{\star}}{a_{\mathrm{end}}^{2} \phi_{\mathrm{end}}} & \gtrsim\left(\frac{M_{0}}{\sqrt{\bar{\alpha}_{0}} T_{0}}\right)\left(1-\delta_{\star}\right)^{-1} \xi^{2} \\
\times & \frac{1}{4 \pi \epsilon|f|\left(g+\frac{c_{2} \theta}{\phi}\right)^{2}\left[1-\frac{2 d_{\star}^{\mathrm{NC}}}{(2 g+1)|f|\left(1-\delta_{\star}\right) a_{\star}^{3} \phi_{\star}}\right]},
\end{aligned}
$$

where the time $t_{\text {end }}$ is devoted to the end of inflation in which the entropy is produced. The subscript 0 stand for the present epoch, $M_{0}$ is the value of the Planck mass today and $\bar{\alpha}_{0}=\gamma\left(t_{0}\right) \eta_{0}=(8 \pi / 3)\left(\pi^{2} / 30\right) \bar{g}\left(t_{0}\right) \eta_{0}$, 


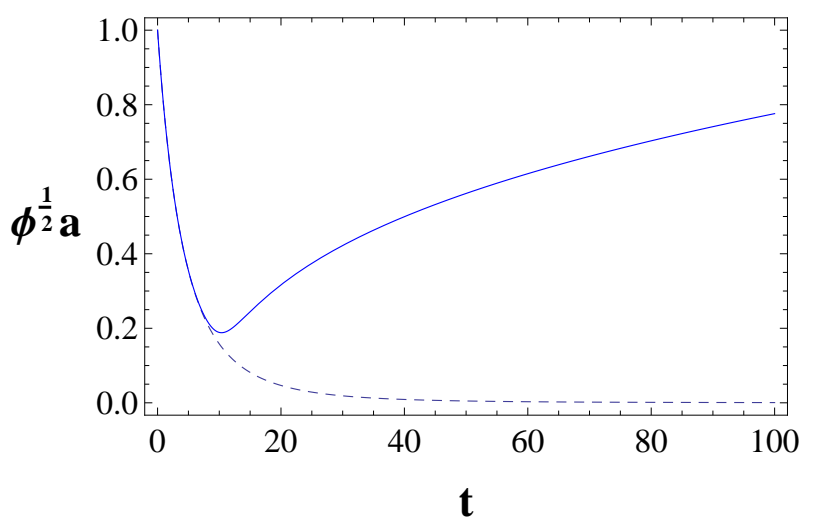

Figure 2: The time behavior of $a \phi^{1 / 2}$ for the commutative case (dashed curve) and NC case (solid curve). The initial conditions are $c_{2}=1=a_{0}, \omega=-1.36$ and $\theta=-0.000001$ for the NC case [4].

in which $\eta_{0}$ is for the ratio today of the energy density in matter to that in radiation. The constraint (23) is the generalized (noncommutative) version of the one resulted in [2] provided the $\mathrm{BD}$ coupling is taken as a constant therein.

We should note that, in the commutative case, the constraint (21), even with assuming a favorite set of initial conditions, implies that the quantity $a^{2} \phi$ decreases with the cosmic time, which also indicates that the Planck mass must decrease during inflation. Consequently, to get an admissible result, a branch change must be induced [2].

In the $\mathrm{NC}$ case, considering the lower sign with $c_{2}>0$ (D branch) and employing (18), (9) and the expression for $g^{ \pm}$, we obtain $d \ln \left(a \phi^{\frac{1}{2}}\right) / d t=$ $-|f| /\left(2 \xi a^{3} \phi\right)\left[\operatorname{sgn}\left(c_{2}\right) \xi+\frac{2 c_{2} \theta}{\phi}\right]$, which, for the commutative case, is reduced to $d \ln \left(a \phi^{\frac{1}{2}}\right) / d t=-|f| /\left(2 a^{3} \phi\right)$. It is easy to show that that, for the commutative case, the quantity $a \phi^{1 / 2}$ always decreases with the cosmic time, see Fig. 2. We should emphasize that such a result is not in agreement with the observational data [4].

For a general $\mathrm{NC}$ case, fortunately, for $c_{2}>0, \theta<0$, and lower case, i.e., the $\mathrm{D}$ branch, we have shown numerically that at the early times, $a \phi^{1 / 2}$ behaves similar to its corresponding in the commutative case. However, after reaching a nonzero minimum, it starts to increase, see Fig. 2.

\section{Conclusions}

In this paper, we have introduced a NC BD setting. Such a scenario bears much resemblance to the settings assumed in NC quantum cosmology or a few classical noncommutative gravitational/cosmological investigations in theories alternative to general relativity $[15,13,12,14])$.
We have constructed a NC generalized BD setting to include key ideas of duality and branch changing as well as gravity-driven acceleration and kinetic inflation. Then, we have assumed that $N=1$ and there is neither a scalar potential nor a cosmological constant. Moreover, we have assumed that the Lagrangian density of the ordinary matter is absent.

In this scenario, we have found that the power-law scale factor of the Universe is not similar to the commutative case, but instead it is generalized to be multiplied with a time-dependent exponential warp factor. Moreover, we have seen that the BD scalar field is not in the form of a simple power function of time (similar to its corresponding commutative case), but instead, it can be obtained from a more complicated differential equation.

For $\theta=0$, we have shown that our herein model yields an extended model of the de Sitter-like space and O'Hanlon-Tupper solutions. In the latter, the integration constants play the role of the duality transformations introduced in the context of the BD theory [11].

For case Ia, we have shown that the scale factor always accelerates in the commutative case, while, for the $\mathrm{NC}$ case, it accelerates only for very early times, and after a very short time, it turns to give a decelerated universe. Consequently, our model can be an appropriate inflationary model, in which we can overcome the graceful exit problem. Moreover, contrary to the commutative model in which the scale factor always accelerates in late times, we get a zero acceleration epoch for the Universe in the $\mathrm{NC}$ case. It has believed that this result can be interpreted as coarsegrained explanation of the quantum gravity footprint.

We have shown that both the nominal and sufficient requirements associated to the inflation can be fully satisfied in our NC model.

In the $\mathrm{BD}$ theory with a variable $\omega[2]$, for a kinetic inflation model, all the accelerations in the $\mathrm{D}$ branch suffer from the graceful exit problem. However, for our NC model, we have shown that his problem is appropriately solved due to the presence of the NC parameter: at the very early times, although, the same as the commutative case, $a \phi^{1 / 2}$ decreases with the cosmic time, while after a very short time, it starts to increase with time, which is in agreement with observations.

Acknowledgements. S. M. M. Rasouli is grateful for the support of Grant No. SFRH/BPD/82479/2011 from the Portuguese Agency Fundação para a Ciência e Tecnologia. He is also extremely grateful to the organizers of the 16-th Gamow International Conference/Summer School, specially, prof. Alexander Zhuk, for their warm hospitality during his attendance at the conference as well as his visiting time. This research work was supported by Grants No. CERN/FP/123618/2011 and No. PEstOE/MAT/UI0212/2014. 


\section{References}

[1] J. J. Levin, Phys. Rev. D 51, 462 (1995).

[2] J. J. Levin, Phys. Rev. D 51, 1536 (1995).

[3] C. Brans and R.H. Dicke, Phys. Rev. 124, 925 (1961).

[4] S.M.M. Rasouli and P.V. Moniz, Phys. Rev. D 90, 083533 (2014).

[5] S.M. M. Rasouli, M. Farhoudi and H. R. Sepangi, Classical Quantum Gravity 28, 155004 (2011); S. M. M. Rasouli, Prog. Math. Rel., Gravit. Cosmol. 60, 371 (2014).

[6] S.M. M. Rasouli, M. Farhoudi and P. V. Moniz, Classical Quantum Gravity 31, 115002 (2014).

[7] V. Faraoni, Cosmology in Scalar Tensor Gravity (Kluwer Academic, Dordrecht, 2004).

[8] R. Brustein and G. Veneziano, Phys. Lett. B 329, 429 (1994).
[9] J. O'Hanlon and B.O.J. Tupper, Nuovo Cimento Soc. Ital. Fis. 7B, 305 (1972);

[10] G. Veneziano, Phys. Lett. B 265, 287 (1991).

[11] J. E. Lidsey, Phys. Rev. D 52, R5407 (1995); Classical Quantum Gravity 13, 2449 (1996).

[12] S. M. M. Rasouli, M. Farhoudi and N. Khosravi, Gen. Rel. Grav. 43, 2895 (2011).

[13] S. M. M. Rasouli, A. H. Ziaie, J. Marto, and P. V. Moniz, Phys. Rev. D 89044028 (2014).

[14] S. M. M. Rasouli, A. H. Ziaie, S. Jalalzadeh and P. V. Moniz, Annals of Phys. 375154 (2016).

[15] W. Guzmán, M. Sabido and J. Socorro, Phys. Lett. B 697, 271 (2011). 